\title{
Supporting Information: Single-Walled Carbon Nanotube Dark Exciton Photoluminescence Dynamics
}

\author{
Trevor M. Tumiel, ${ }^{1}$ Mitesh Amin, ${ }^{2}$ and Todd D. Krauss ${ }^{1,2 *}$ \\ ${ }^{1}$ Department of Chemistry and ${ }^{2}$ The Institute of Optics, University of Rochester, Hutchison Hall, \\ Box 270216, Rochester, NY 14627, United States
}

t.tumiel@rochester.edu,mamin@ur.rochester.edu,tkrauss@ur.rochester.edu*

\section{Table of Contents}

1. PLE map acquired for a sample of $(6,5)$-enriched SWCNTs...................... S2

2. Absorbance spectra for samples prepared with various probe sonication times.........

3. Raman spectra for $(6,5)$-enriched SWCNTs samples............................. S4

4. Absorbance and PL spectra for a $(7,5)$-enriched SWCNT sample................... S5

5. Lifetime measurements for $(7,5)$-enriched SWCNTs........................... S6

6. Summary plots of the average $\mathrm{E}_{11}$ and $\mathrm{KDE}$ state lifetime values for $(6,5)$ SWCNTs..

7. Rate equations used for kinetic modeling of KDE time decays................... S8 


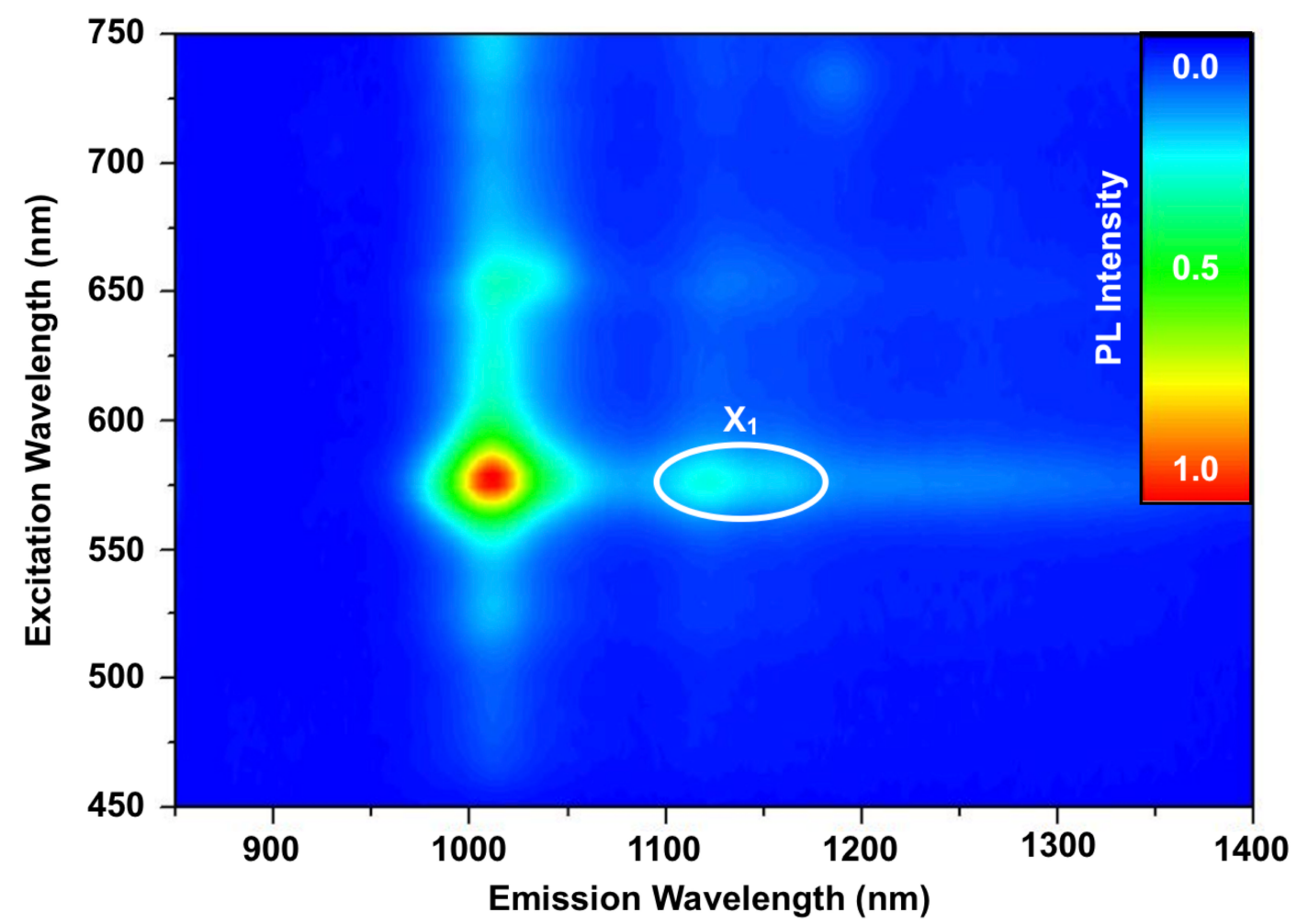

Figure S1. Photoluminescence excitation (PLE) map for a (6,5)-enriched CoMoCAT SWCNT sample probe sonicated for 4 hours (see Experimental Methods), demonstrating exceptional enrichment in a single NT species. Emission corresponding to the $\mathrm{X}_{1}$ feature is labeled. 


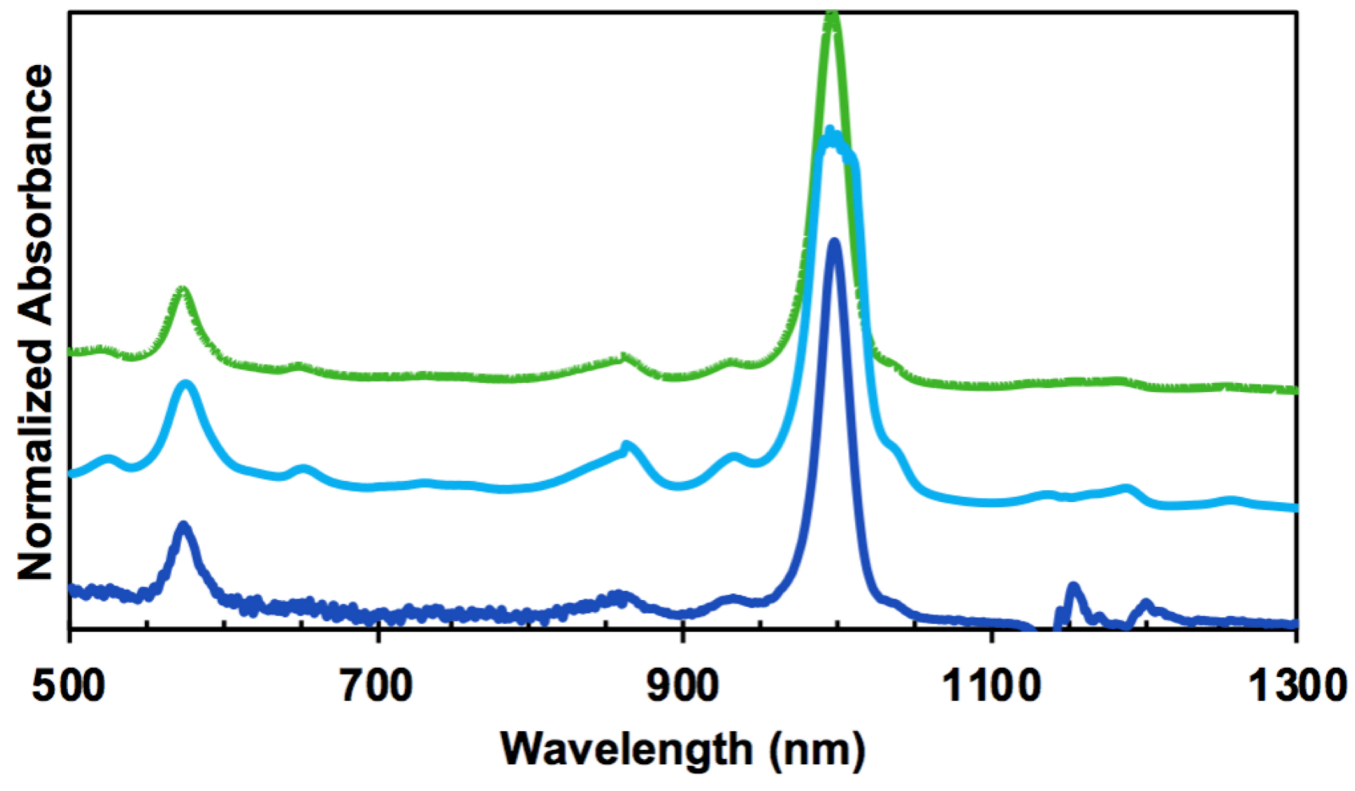

Figure S2. Absorbance spectra for (6,5)-enriched SWCNT samples probe sonicated for 4 (blue), 20 (aqua) and 50 (green) hours. 


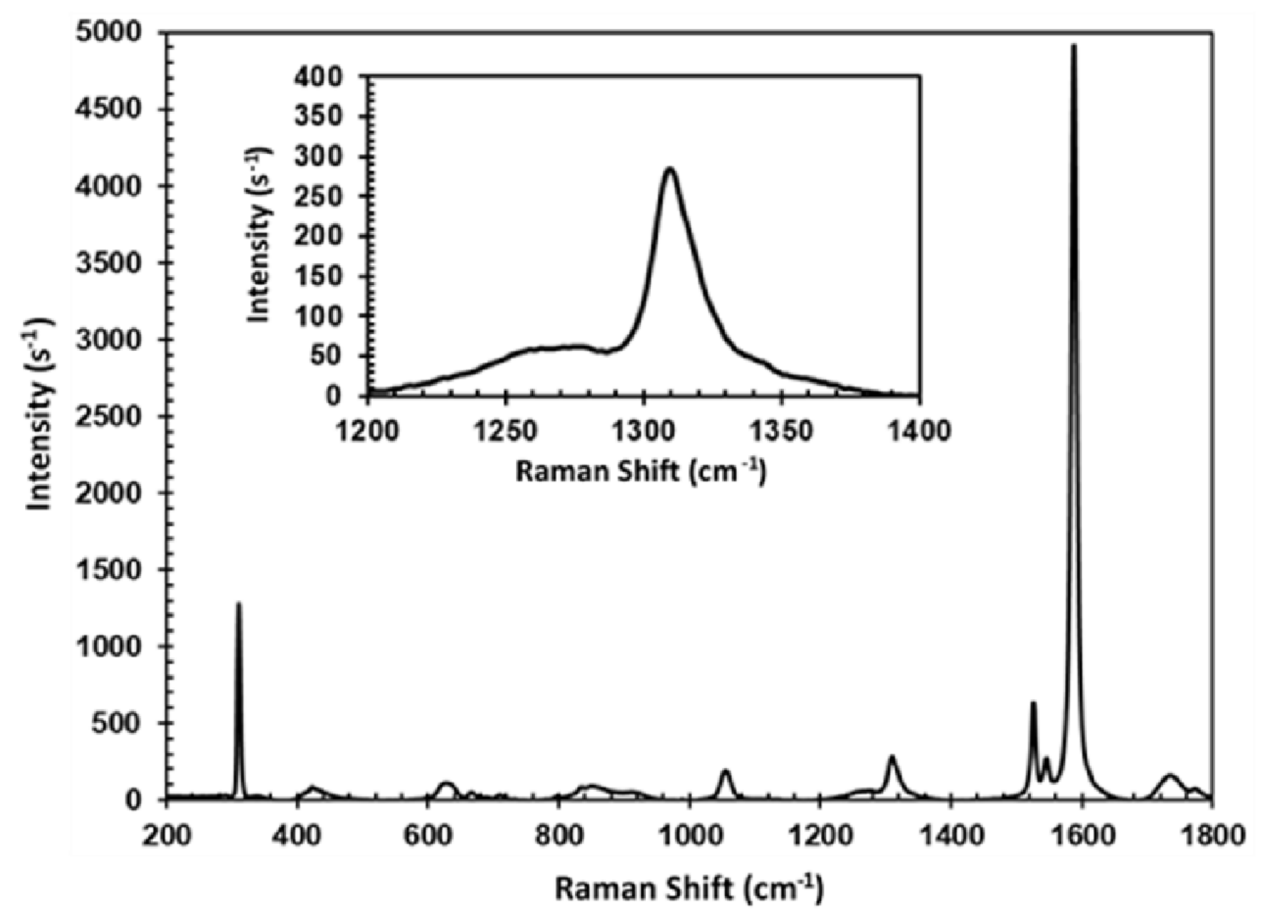

Figure S3. Raman spectrum of a $(6,5)$ SWCNT sample probe sonicated for 20 hours (see Experimental Methods section for experimental details pertaining to Raman measurements). The inset displays the Dband, a Raman feature that corresponds with strong elastic scattering from a defect coupled with an $\mathrm{A}_{1}$ ' iTO phonon. The most intense peak of the D-band $\left(\sim 1310 \mathrm{~cm}^{-1}, \sim 163 \mathrm{meV}\right)$ matches the energy difference between the KDE state and $\mathrm{X}_{1}$ virtual state, as expected. Because the KDE state is situated roughly 30 meV above the $E_{11}$ state, we would expect the energy difference between the $E_{11}$ and $X_{1} P L$ features to be $\sim 130 \mathrm{meV}$, which matches our experimental data very well. The G-mode $\left(\sim 1590 \mathrm{~cm}^{-1}\right)$ is often compared to the D-band to ascertain the defective nature of the SWCNTs being studied. It is clear from the Raman spectrum that the defect density is low, as the D-band intensity is significantly lower than the G-mode intensity. Note that the Raman spectrum collected for $(6,5)$ SWCNT samples probe sonicated for 4 and 50 hours displayed similar D-band to G-band intensity ratios. 

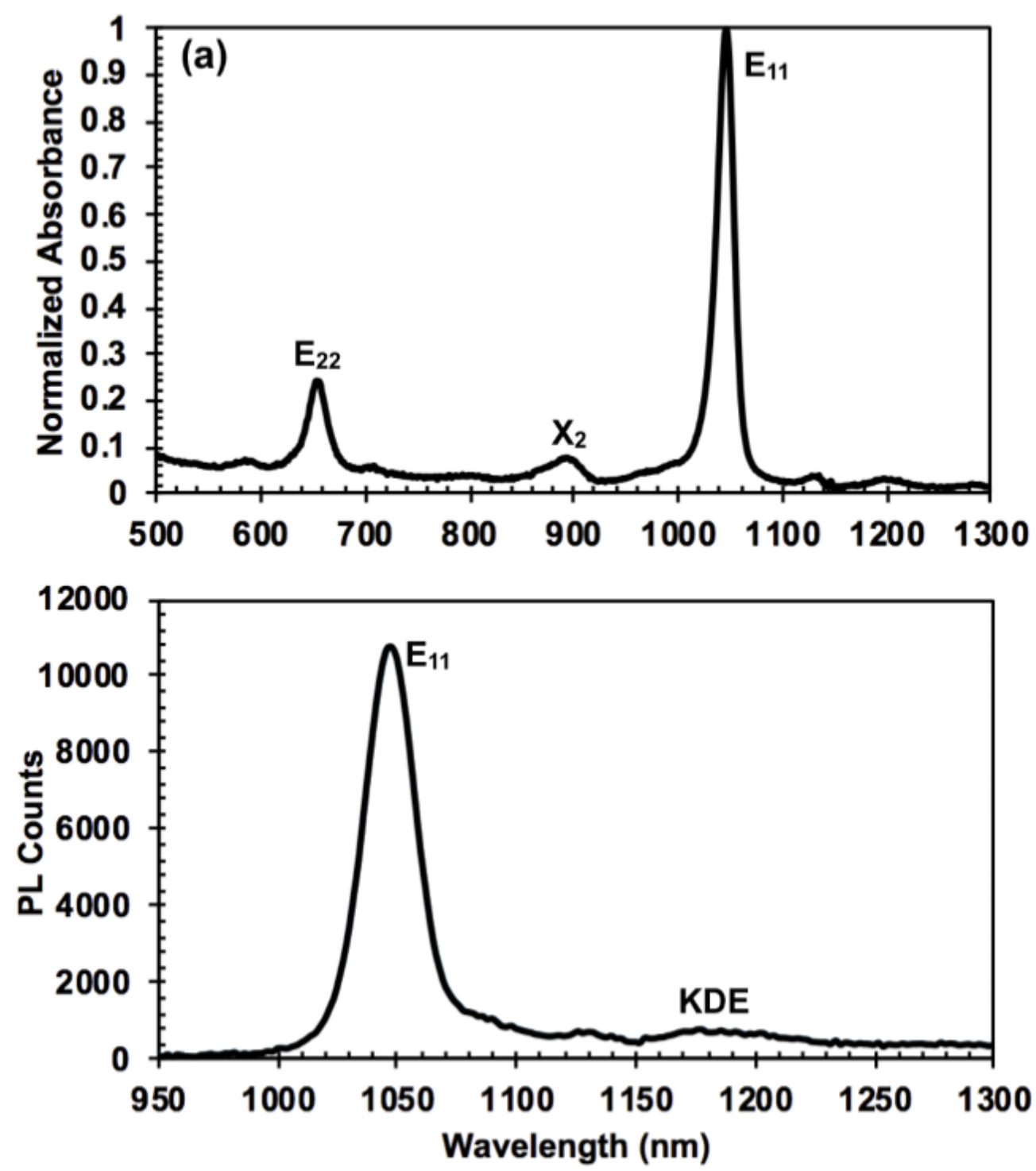

Figure S4. To confirm that the KDE PL dynamics discussed in the main manuscript text are applicable across multiple chiralities, $(7,5)$ SWCNTs were studies in addition to the $(6,5)$ SWCNTs. (a) Absorbance and (b) ensemble PL spectra of $(7,5)$-enriched SWCNT suspensions probe sonicated for 4 hours are largely free of additional chiralities or impurities. Notable features in the absorbance spectrum unique to $(7,5)$ SWCNTs have been labeled $\left(E_{11}, E_{22}, X_{2}\right)$. The very minor absorbance features at 1130 $\mathrm{nm}$ and $1195 \mathrm{~nm}$ are most likely E 11 features corresponding to $(7,6)$ and $(8,6)$ SWCNTs, respectively. Notable features in the ensemble PL spectrum have also been labeled ( $\mathrm{E}_{11}$, $\mathrm{X}_{1}$ ). 

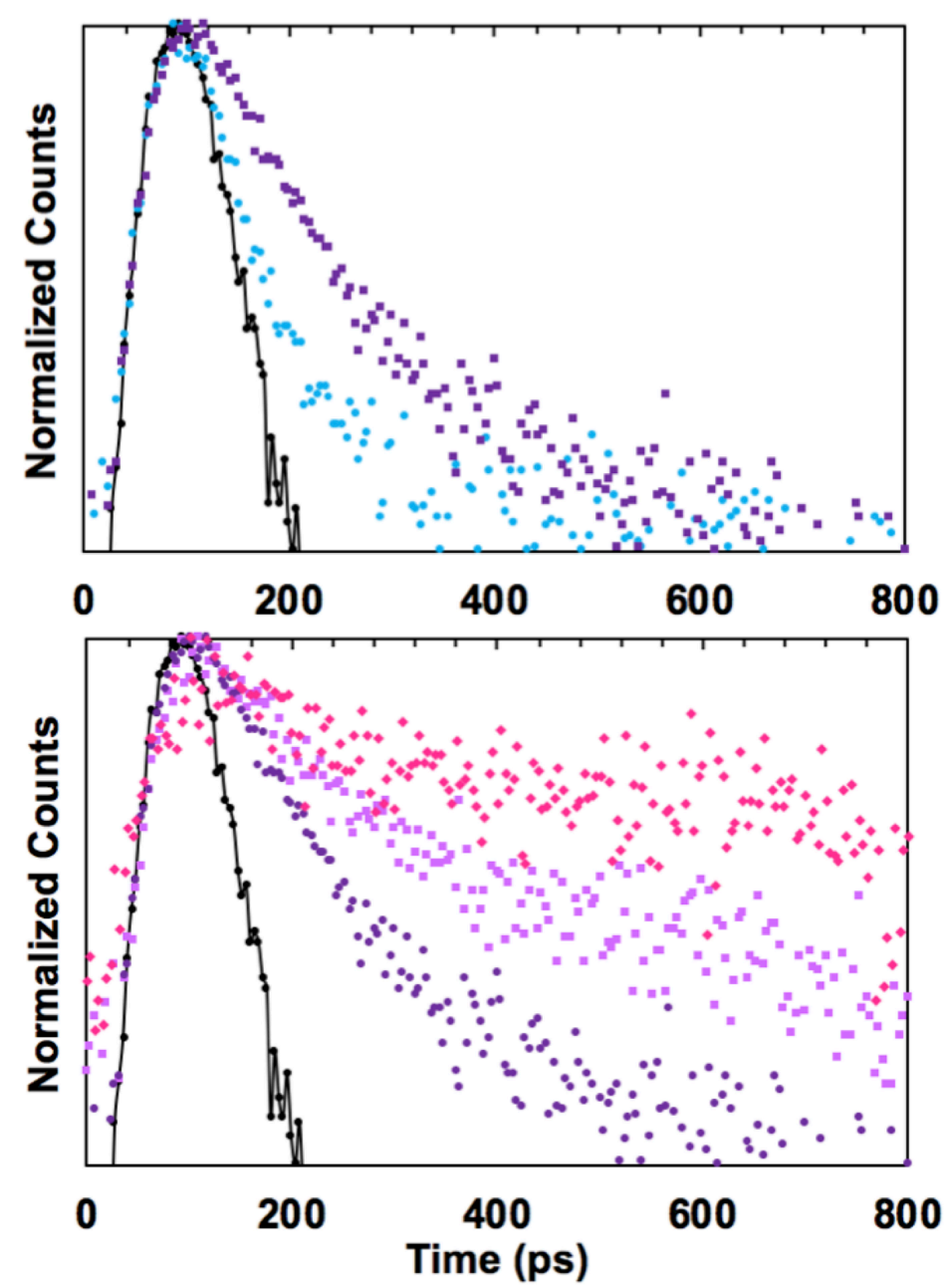

Figure S5. (Top panel) Room-temperature time decays for the $\mathrm{E}_{11}$ state (aqua) and KDE state (purple) for a $(7,5)$-enriched SWCNT sample probe sonicated for 4 hours. The instrument response function is outlined in black. It is clear from these time decays that the average lifetime of the KDE state is longer than that of the $E_{11}$ state, which is consistent with observations made for $(6,5)$ SWCNTs. (Bottom panel) Time decays for the KDE state at $293 \mathrm{~K}$ (purple), $200 \mathrm{~K}$ (violet) and $100 \mathrm{~K}$ (pink) for the same (7,5)enriched SWCNT sample probe sonicated for 4 hours. The instrument response function is outlined in black. As the temperature decreases, the average lifetime of the KDE state increases, a trend that is consistent with our analysis of KDE PL dynamics of $(6,5)$ SWCNTs. Specifically, lower temperatures cause increased thermalization to the energetically lower-lying $\mathrm{D}_{11}$ state that ultimately leads to increased bright-dark state mixing and, thus, longer PL lifetimes. Note that, at sufficiently low temperatures, oscillations in the bias voltage due to the ringing of the detector's gate-on window begin to appear in the time decays (pink decay, $\sim 600 \mathrm{ps}$ ) due to a decrease in PL photon counts. 




Figure S6. Plots of the average lifetime values for the $\mathrm{E}_{11}$ (aqua) and KDE (purple) states versus probe sonication time at room- and low-temperature for $(6,5)$ SWCNTs. That data displayed in these plots are identical to the average lifetime data presented in Table 1. As probe sonication time increases, which effectively increases the SWCNT lattice defect concentration, the average lifetimes of both the $\mathrm{E}_{11}$ and KDE states increase in a similar fashion. At low temperature, the remarkable similarity between the increasing trend of $E_{11}$ and $\mathrm{KDE}$ state average lifetime values with increasing probe sonication time suggests that the photophysical processes dominating $\mathrm{E}_{11}$ exciton dynamics are identical to those dominating KDE exciton dynamics. Specifically, the similar trends suggest that mixing between the bright and dark singlet excitonic states, which is accentuated at low temperatures and higher lattice defect concentrations, plays a significant role in $\mathrm{KDE}$ exciton dynamics just as it does in $\mathrm{E}_{11}$ exciton dynamics. 




Figure S7. Rate equations used to describe the KDE time decay, emphasizing the mixing between the $\mathrm{E}_{11}$ and $\mathrm{D}_{11}$ states. The rate equations were modeled after those used by Ishii et al. to study the dark-to-bright exciton conversion in SWCNTs. 